\title{
POSITION-SCANNING SPECTROPHOTOMETER AS A MEANS OF OBSERVING MULTICOMPONENT DIFFUSION PHENOMENA IN LIQUID PHASE
}

\author{
Wataru EGUCHI, MaKoto HARADA, MotonaRi ADACHI \\ MASATAKA TANIGAKI AND KAZUO KONDO \\ Institute of Atomic Energy, Kyoto University, Uji 611
}

Key Words: Mass Transfer, Spectrophotometer, Multicomponent Diffusion Coefficients, Chemical Reaction, Nickel Chloride, Cobalt Chloride, Nitroso R Salt, Copper Sulfate

\begin{abstract}
A new device, called the position-scanning spectrophotometer, which measures the concentration profiles of lightabsorbing solute species in the course of an unsteady multicomponent diffusion process, is described. Illustrative examples of application of this spectrophotometer to the coupled ternary diffusion process in the water- $\mathrm{NiCl}_{2}-\mathrm{CoCl}_{2}$ system and to diffusion-controlled mass transfer with an irreversible instantaneous chemical reaction between nitroso- $\mathrm{R}$ salt and copper sulfate are shown. These examples indicate that this spectrophotometer is a powerful means of studying complex diffusional processes in liquid phase.
\end{abstract}

\section{Introduction}

Diffusion of more than a single solute species often occurs simultaneously in the operation of mass transfer processes. The flux of each solute is influenced by the diffusion of other components, the existing electrostatic potential and the chemical reactions occurring in the liquid phase. For a basic understanding of the complicated diffusion phenomena, it is important to measure the concentration profiles of more than one solute species in the diffusion layer.

A number of techniques have been developed for this purpose. Methods which directly measure the concentrations of samples obtained after diffusion has progressed for a certain period of time, such as the shear cell method and the solidification method, are most basic. The profiles measured by these methods, however, may be different from those in the actual diffusion process, due to the errors anticipated in the rotation procedure of the cells or in the solidifying process. Furthermore, concentration profiles at different diffusion periods can only be obtained from separate experiments.

Methods which utilize the refractive index or its gradient are excellent means of obtaining information on the concentration profiles of solute species in the course of unsteady diffusion occurring in lightpermeable liquids. Among these, the Gouy interferometer is most often used in the measurement of diffusion coefficients in multicomponent systems. ${ }^{2)}$

Received November 28, 1983. Correspondence concerning this article should be addressed to M. Tanigaki. W. Eguchi and M. Tanigaki are now at Dept. of Chem. Eng., Kyoto Univ., Kyoto 606. K. Kondo is now with Sumitomo Metal Ind. Ltd., Amagasaki, 660.
Other methods, such as the Mach-Zehnder and Rayleigh interferometers and the Schlieren cylindrical lens method, have also been used. Even though there is no doubt about the superior nature of these methods on account of their high positional resolution, they are of limited use when applied to multicomponent systems because only a single kind of information, i.e., refractive index, is available.

Many solute species have their own characteristic light absorption and this can be used for the measurement of solute concentration profiles. Linhart" employed the technique of recording the intensity profiles of light beams irradiated onto the cell uniformly and transmitted through the liquid phase in the cell onto a photosensitive plate. The use of a self-scanning image sensor which consists of one-dimensional array of small photosensitive elements was tried by Kamiwano et al. ${ }^{6)}$ as a substitute for the photosensitive plate. High precision in intensity measurement, however, cannot be expected by these methods due to the lack of uniformity in the light beam and in the sensor sensibility.

Measurement of the intensity profile is also possible if either the spectrophotometer ${ }^{5)}$ or the cell ${ }^{3)}$ is moved relative to each other. Rapid positional scanning required in the case of unsteady diffusion is not possible by these methods, due to disturbances that may be brought about by the mechanical movement.

The authors have developed a device in which a slitlike horizontal light beam is made to scan vertically the fixed mass transfer cell by means of an oscillating mirror arrangement. Rapid scan over the diffusion layer in the cell is possible with this device, enabling 
the measurement of absorbance profiles during unsteady diffusion. Measured absorbance profiles at different wavelengths make possible the separate determination of concentration profiles of the diffusing species in a multicomponent system.

This report describes this new device, called the position-scanning spectrophotometer, and the procedure employed. Examples of the observation of multicomponent diffusion phenomena are also given.

\section{Position-Scanning Spectrophotometer}

The constitution of the position-scanning spectrophotometer (called PSS hereafter) is shown in Fig. 1. The basic part of the optical system was from a commercial spectrophotometer (Model SM401, manufactured by Union Giken Co., Osaka, Japan). The monochromatic light beam (normally $50 \mu \mathrm{m}$ in width) from the narrow vertical slit, $S_{2}$, is incident upon the surface of the oscillating camera mirror, $\mathrm{OM}$, by means of a collimating mirror, $\mathrm{CM}$. The focal lengths of these two mirrors are both $400 \mathrm{~mm}$. The oscillating mirror driven by the computer-controlled stepping motor, $\mathrm{P}_{2}$, swings the light beam horizontally in the narrow region shown by the broken lines in Fig. 1. The vertical-to-horizontal light converter, $\mathrm{VH}$, then converts the horizontal swing of the vertical slit-like light to the vertical movement of the horizontal one, allowing vertical position-scanning at the mass transfer cell, TC, placed at the focal plane of the light beam. The scan range is in total $20 \mathrm{~mm}: 10 \mathrm{~mm}$ upward and downward from the center of the cell. The light beam transmitted through the mass transfer cell is focussed nearly at the center surface of the headon photomultiplier tube, $\mathrm{PM}_{1}$, by means of the quartz lens, $\mathrm{LS}_{1}$.

Because of the single-beam measurement employed in the present system, any fluctuations in the intensity of the light source give rise to an instability in the output voltage of the photomultiplier. To avoid this, part of the light beam from the slit, $\mathrm{S}_{2}$, is introduced to another photomultiplier, $\mathrm{PM}_{2}$, by the beam splitter, SP, and the high voltage applied to both photomultipliers is adjusted so that the output signal of $\mathrm{PM}_{2}$ holds a constant level throughout the measuring period. Measurements using light of any wavelength over the range of $200-1000 \mathrm{~nm}$ can be carried out automatically through the movement of the grating, $\mathrm{G}$, driven by another computer-controlled stepping motor, $\mathrm{P}_{1}$, with the exception of the manual switchover between the iodine lamp and the deuterium discharge tube.

Absorbance measurements at multi-wavelengths at predetermined times are carried out fully automatically by the computer according to the schedule described below. When the timing counter issues the

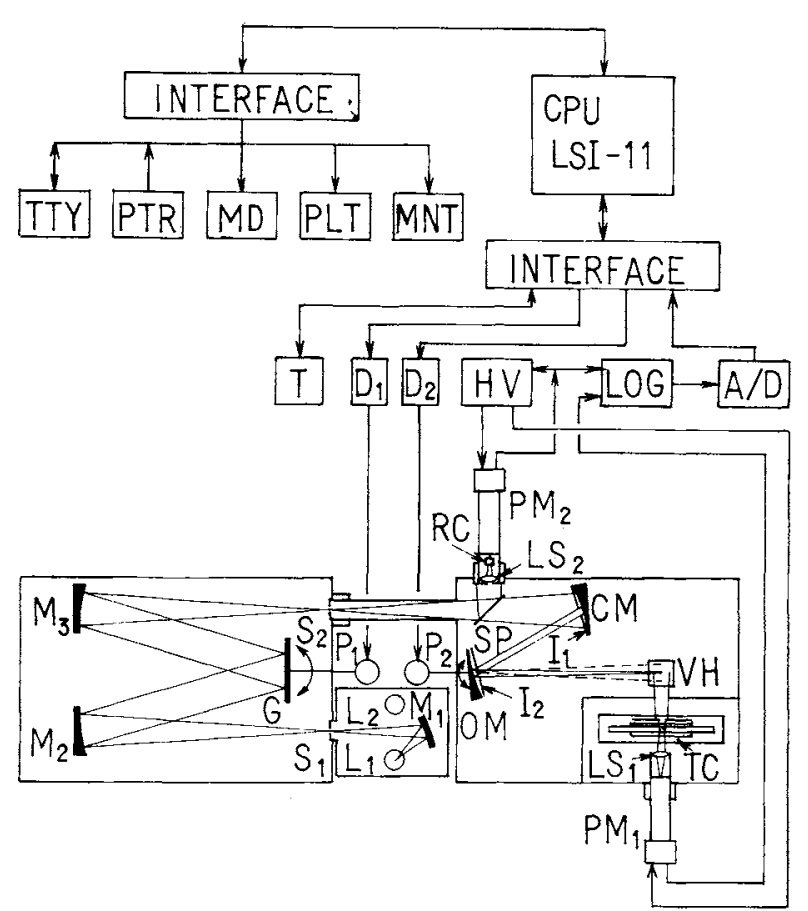

Fig. 1. Constitution of PSS. A/D: analog-to-digital converter; CM: collimating mirror; $\mathrm{D}_{1}, \mathrm{D}_{2}$ : stepping motor drive; G: grating; $H V$ : high voltage; $I_{1}, I_{2}$ : iris; $\mathbf{L}_{1}, \mathbf{L}_{2}$ : light source; LOG: logarithmic transformer; $\mathrm{LS}_{1}, \mathrm{LS}_{2}$ : focussing lens; $\mathrm{M}_{1}$, $\mathrm{M}_{2}, \mathrm{M}_{3}$ : mirror; $\mathrm{MD}$ : magnetic disk; MNT: monitor; OM: oscillating camera mirror; $\mathrm{P}_{1}, \mathrm{P}_{2}$ : stepping motor; $\mathrm{PM}_{1}, \mathrm{PM}_{2}$ : photomultiplier tube; PLT: plotter; PTR: paper tape reader; RC: reference cell; $S_{1}, S_{2}$ : slit; SP: beam splitter; $T$ : timer; TC: mass transfer cell; TTY: graphic typewriter; VH: vertical-tohorizontal light converter.

instruction to start a set of measurements at the first predetermined time, the output signal voltage of the photomultiplier at the first wavelength and at the uppermost position in the mass transfer cell is converted into the digital signal by $\mathrm{A} / \mathrm{D}$ converter, subtracted the corresponding reference absorbance (which will be discussed later), and is stored in the internal memory register of the computer. Then the stepping motor, $\mathrm{P}_{2}$, advances a step and measurement is carried out at this new position as before. This procedure is repeated 256 times until measurement at the lower end (final) position is completed. This position-scanning measurement takes $2-4 \mathrm{~s}$.

After the driving back of the oscillating mirror to the uppermost position, the stepping motor, $\mathrm{P}_{1}$, is signaled to change the wavelength up to the second preset value, where the same measurements as at the first wavelength are carried out. The maximum speed of change in wavelength is $25 \mathrm{~nm} / \mathrm{s}$. This set of measurements is repeated at different wavelengths up to the final designated one. After the return of the position of the oscillating mirror to the uppermost position at the final wavelength, the stepping motor, $\mathrm{P}_{1}$, drives the grating back to the first wavelength and PSS is in the awaiting mode, waiting for the start 
signal at the second preset time.

Time required from the measurement at the uppermost position for the first wavelength to that at the lowermost position for the final wavelength is usually in the range of 4 to $100 \mathrm{~s}$, depending on the number and range of wavelengths used, speed of position scanning and of the change in wavelength, and the number of repetitive measurements at each position. Fortunately, diffusion is a slowly occurring phenomena and the above time period can be neglected in measurements at times later than 3 to 30 minutes.

The angle of incident light beam to the mass transfer cell is different for different positions due to the optical system used. Further, the position on the photomultiplier surface where the transmitted light beam focusses is not exactly the same, even with the use of the light-collecting lens, LS $_{1}$. These two factors make the output signals differ at different positions as well as at different wavelengths. To correct these differences, a set of reference absorbance measurements are carried out with the mass transfer cell empty or filled with a reference liquid prior to the experimental procedure explained above.

\section{Examples of Measurements Using PSS}

\subsection{Multicomponent diffusion in liquid phase}

In a multicomponent system, diffusion of a solute component occurs not only due to its own concentration gradient, but also to those of other components. This multicomponent diffusion is important in the analysis of diffusional operations and also for the understanding of the solute-solute and solutesolvent interactions in liquid phases. The measurement of these multicomponent diffusion coefficients reported in the literature is limited, because of either difficult experimental procedure or complicated data analysis. ${ }^{2)}$ With PSS, the determination of multicomponent diffusion coefficients of light-absorbing solutes in liquid can be carried out easily without complicated data analysis, because the concentration profiles of the constituting solute species in the course of unsteady diffusion process are directly measured. An example of measurement in the water- $\mathrm{NiCl}_{2}-$ $\mathrm{CoCl}_{2}$ ternary system at $298 \mathrm{~K}$ is presented in this section.

The two solutes, $\mathrm{NiCl}_{2}$ and $\mathrm{CoCl}_{2}$, have their maximum light absorption at 394 and $511 \mathrm{~nm}$, respectively, with small interfering absorption by the other solute. This makes the system quite adequate for the present illustrative purpose. It was confirmed that the Beer-Lambert law is valid and that the absorbance of a mixture solution is expressed as linear combinations of the two solutes.

The slide contact cell used is shown in Fig. 2. It is basically the same as that developed by Neurath. ${ }^{8}$ A fixed lower spacer made of Pyrex glass with an opening $\mathrm{A}$ and a slide spacer with openings $\mathrm{B}$ and $\mathrm{C}$ are tightly pressed to each other between two quartz plates, forming the mass transfer cell. The scan range is shown by the broken line in compartments A and B in Fig. 2.

With compartment B set on top of $A$, the reference liquid is introduced from the small hole at the top of compartment B. With the cell placed in PSS, the liquid temperature is equilibrated with the constanttemperature water at $298 \pm 0.1 \mathrm{~K}$ which is circulating through the cell holder. The reference data taken with this setting are stored in the computer so that they can be used to correct the absorbance data afterwards.

Then the liquid in compartments $\mathrm{A}$ and $\mathrm{B}$ is replaced with the lighter liquid used for the diffusion experiment. Now compartment $\mathrm{C}$ of the slide spacer is made to come on top of A by rotation of the handle, and the heavier liquid is introduced in compartments $\mathrm{A}$ and $\mathrm{C}$, after the removal of the lighter liquid in compartment A. After the duration of time required for temperature equilibration again, the handle is reversely rotated. This makes possible the contact of the lighter liquid in compartment B with the heavier one in compartment $\mathrm{A}$, forming a step-wise initial concentration profile at the sliding surface. This is the start of the diffusion experiment and the initiation

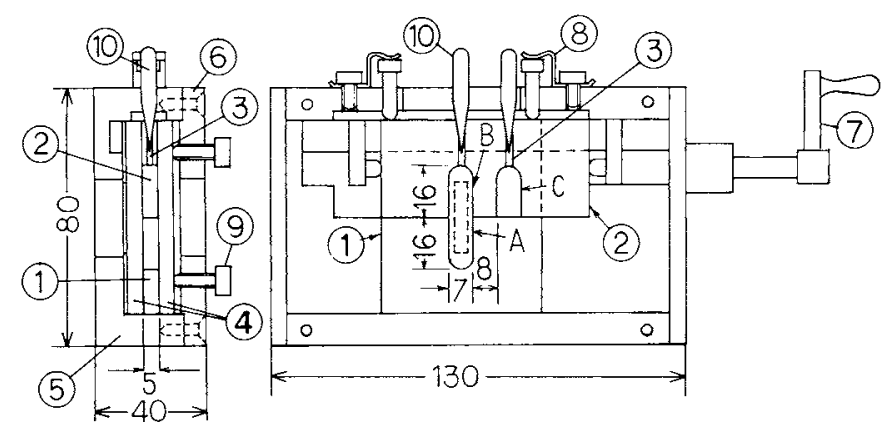

Fig. 2. Slide contact cell. (1): fixed Pyrex glass spacer with opening A (2): slide spacer with openings $B$ and C (3): liquid supply hole (1 mm in diameter) (4): quartz plates (5): brass cell holder (6): brass lid (7): handle (8): spring mechanism to press the slide spacer tightly to the fixed spacer(9): Screw used to press the quartz plates and the spacers (10): plug made of polyfluoroethylene resin. All dimensions are in millimeters. 

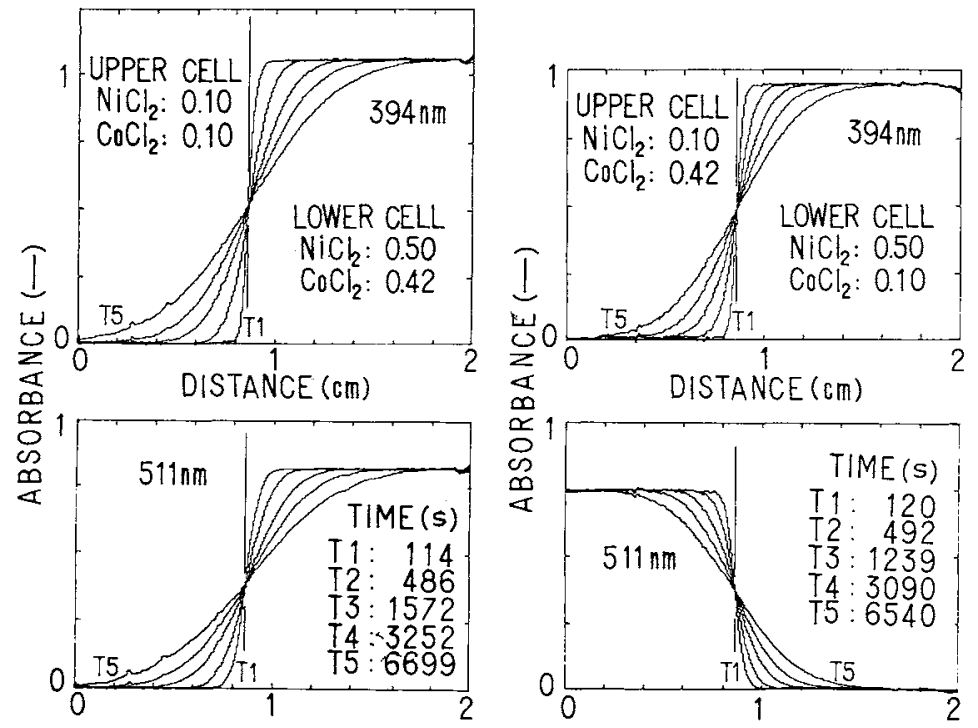

Fig. 3. Original absorbance profiles in water $-\mathrm{NiCl}_{2}-\mathrm{CoCl}_{2}$ system at $298 \mathrm{~K}$. Concentrations of solutes are in $\mathrm{kmol} / \mathrm{m}^{3}$. An aqueous solution of $\mathrm{NiCl}_{2}: 0.10 \mathrm{kmol} / \mathrm{m}^{3}$ and $\mathrm{CoCl}_{2}: 0.10 \mathrm{kmol} / \mathrm{m}^{3}$ was used as the reference liquid in both experiments. Times of measurements shown in the figure are the average values of those at the two wavelengths.

signal to the computer starts the automatic sets of absorbance measurements according to the procedure stated in the previous section.

The original data plotted by the graphic typewriter are shown in Fig. 3. The set of data on the left-hand side are for the case of diffusion of two solutes in the same direction. The distance in the figure corresponds to that downward from the uppermost position. From the curves at the earliest time, it can be seen that nearly step-wise initial concentration profiles are realized with the present slide contact cell. The vertical lines near the center show the slide plane. The fact that smooth curves at different times cross each other at this slide plane indicates the occurrence of molecular diffusion without disturbances in the liquid phase. Curves on the right-hand side in Fig. 3 are for the case of diffusion of two solutes in opposite directions.

Diffusion in the above three-component system is governed by the following generalized Fick's law:

$$
\begin{aligned}
& \frac{\partial c_{1}}{\partial t}=\frac{\partial}{\partial x}\left(D_{11} \frac{\partial c_{1}}{\partial x}\right)+\frac{\partial}{\partial x}\left(D_{12} \frac{\partial c_{2}}{\partial x}\right) \\
& \frac{\partial c_{2}}{\partial t}=\frac{\partial}{\partial x}\left(D_{21} \frac{\partial c_{1}}{\partial x}\right)+\frac{\partial}{\partial x}\left(D_{22} \frac{\partial c_{2}}{\partial x}\right)
\end{aligned}
$$

With the semi-infinite boundary and the step-wise initial conditions, the above equations can then be transformed into ordinary differential equations by the use of the Boltzmann factor $\eta=x / \sqrt{t}$. They then require that the concentration profiles at different times should coincide with each other if molecular diffusion proceeded.

The calculated normalized concentration profiles of the two solutes are plotted as functions of $\eta$ in Fig. 4 . Curves 1 and 2 show the profiles of $\mathrm{NiCl}_{2}$ and $\mathrm{CoCl}_{2}$, respectively, for diffusion in the same direction. Curves 3 and 4 are those for opposite diffusion. The fact that the data for different times lie on a single curve indicates the occurrence of molecular diffusion.

The difference in the curves for both solutes between the diffusions of different directions indicates the non-negligible values of the cross-diffusion coefficients resulting from the solute-solute interaction in the liquid phase. The reduction of the four diffusion coefficients from the data shown above is presented elsewhere. ${ }^{9)}$

The method of obtaining the four diffusion coefficients using the Gouy interferometer has been highly developed by Gösting et al. ${ }^{4)}$ The high precision in the measurement of fringe positions made pseudo-binary analysis possible. In the present method, on the other hand, concentrations of the two solutes can directly be measured. This makes the method of reducing diffusion coefficients much more direct. ${ }^{9)}$ This is an important advantage when there are more than two solutes present in the system. ${ }^{10)}$

\subsection{Observation of mass transfer phenomena accom-} panied by chemical reaction in aqueous phase

A sample observation of the diffusion process accompanied by a chemical reaction is presented in this section. The system chosen is the following complex-forming reaction between copper sulfate and nitroso-R salt in aqueous phase at $298 \mathrm{~K}$ :

$$
\mathrm{C}_{10} \mathrm{H}_{4}(\mathrm{NO})(\mathrm{OH})\left(\mathrm{SO}_{3} \mathrm{Na}\right)_{2}+\mathrm{CuSO}_{4} \rightarrow \text { complex }
$$

The three wavelengths used were 373, 482 and $804 \mathrm{~nm}$. At the first wavelength, $373 \mathrm{~nm}$, the contri- 


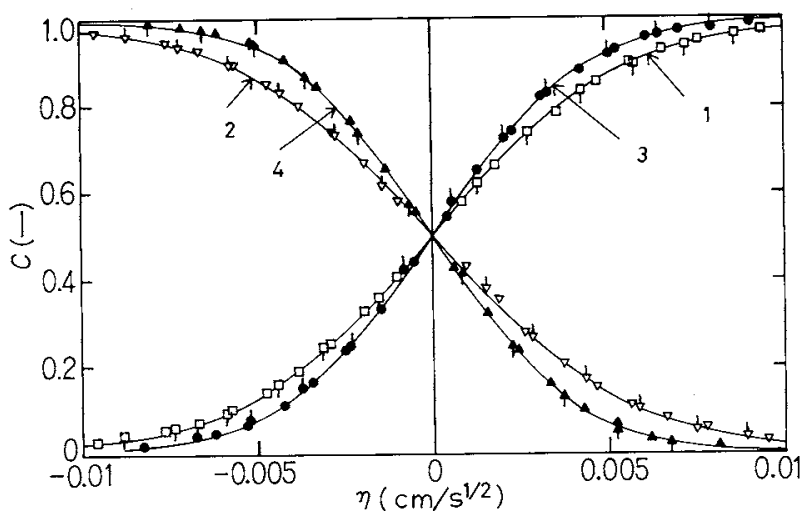

Fig. 4. Normalized concentration profiles in water- $-\mathrm{NiCl}_{2}-$ $\mathrm{CoCl}_{2}$ system. Curve (2) is plotted on the opposite side of the axis $\eta=0$, for visual convenience.

butions of nitroso- $\mathrm{R}$ salt and the complex are comparative. The absorbancies at $482 \mathrm{~nm}$ are mainly those of the complex, though the contribution of Nitroso-R salt cannot be neglected. The absorbancies at $804 \mathrm{~nm}$ are solely due to copper sulfate.

The cell used is the modified flow junction cell developed by Coulson et al. ${ }^{1 \prime}$ and is shown in Fig. 5. The cell spacer, made of polytetrafluoroethylene resin, has an opening which is sandwiched and glued between two quartz plates, forming the transfer cell. The lighter liquid (aqueous solution of nitroso-R salt: $1.99 \times 10^{-4} \mathrm{kmol} / \mathrm{m}^{3}$ ) and the heavier one (aqueous solution of copper sulfate: $8.02 \times 10^{-2} \mathrm{kmol} / \mathrm{m}^{3}$ ) are introduced at the same flow rate from the top and bottom inlet tubes, respectively. The forced exit flow of liquids from the narrow horizontal outlet slits at both sides of the cell eventually makes a step-wise contact of the two liquids at this level possible. After the sharpening of the boundary, the flow of the two liquids is stopped by operating the four cocks of the inlet and the outlet flow lines at the same time. This is the start of measurement.

The resulting absorbance profiles are shown in Fig. 6. The reference data with pure water filled in the cell have been subtracted. The vertical lines near the center show the plane of initial contact. The maximum absorbance level at $482 \mathrm{~nm}$ in Fig. 6 is approximately constant and moves toward the left side in the figure (or into phase of notroso-R salt) as time proceeds. The width of the absorbance profiles, at the same time, increases with a steep curve on the left side and a much less steep one on the right side. In Fig. 6(c), penetration of copper sulfate is seen up to the position of maximum absorption in (b). The partial lowering in the curves in (a) also occurs at this position.

The concentrations of nitroso-R salt (A), copper sulfate (B) and of the complex (C), calculated from the above absorbances at times later than $T_{4}$, are plotted against $\eta$ in Fig. 7. The coincidence of curves

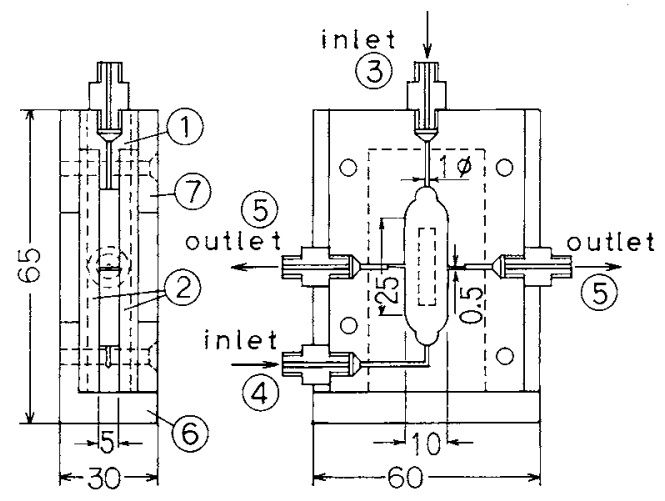

Fig. 5. Flow junction cell. (1): cell spacer made of polytetrafluoroethylene resin (2): quartz plates (3): lighter liquid inlet (4): heavier liquid inlet (5): liquid outlet slits $(0.5 \mathrm{~mm}$ in height and $5 \mathrm{~mm}$ in width) (6): brass cell holder (7): brass lid.
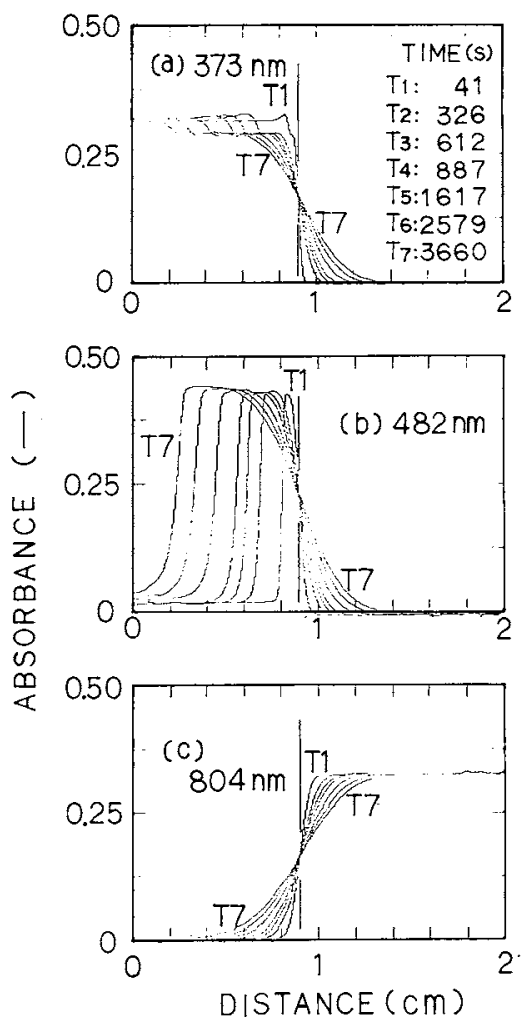

Fig. 6. Original absorbance profiles for the system of nitroso-R salt and copper sulfate at $298 \mathrm{~K}$.

at different times for each species shows that the mass transfer process occurring in this case is controlled by diffusion. At the plane $\eta=\eta_{r}$ (shown by the broken vertical line in the figure), the concentrations of both nitroso-R salt and copper sulfate decrease to zero. The concentration of the complex has the maximum value at this position, with completely different shape of curves on both sides. This indicates the existence of a well-defined reaction plane at $\eta=\eta_{r}$, which moves toward the phase of nitroso- $\mathrm{R}$ salt at a rate proportional to $t^{1 / 2}$. From the observation stated above, it can be concluded that the phenomenon observed 


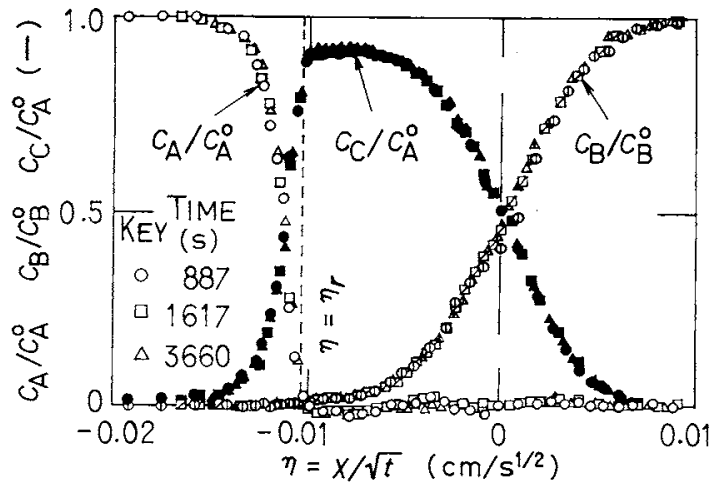

Fig. 7. Normalized concentration profiles for the system of nitroso-R salt and copper sulfate; Superscript, ${ }^{\circ}$, refers to the initial concentrations. The solid vertical line at $\eta=0$ indicates the original contact plane and the broken line shows the reaction plane at $\eta=\eta_{r}$.

here is a diffusion-controlled process accompanied by an irreversible instantaneous reaction.

The information provided by various interferometers is only the profile of the refractive index or its gradient, which is a linear combination of the participating solute concentrations. In this case the mechanism of the reactions occurring in the liquid phase has to be assumed a priori. The profile of the refractive index or its gradient calculated with the assumed reactions can then be compared with the observed one. With PSS, on the other hand, the reaction mechanism occurring in the liquid phase can directly be reduced from the observed solute concentrations.

\section{Conclusions}

A new type of spectrophotometer, called the position-scanning spectrophotometer makes possible the observation of concentration profiles of lightabsorbing solute species in the course of the unsteady multicomponent diffusion process. It is a powerful means of measuring the multicomponent diffusion coefficients and of studying the complex diffusional processes in liquid phase.

\section{Acknowledgments}

The development of the position-scanning spectrophotometer was undertaken with the aid of a portion of a supplementary budget from the Ministry of Education, Japan for application for a raise in special funds for equipment over the fiscal years 1975 and 1978. These grants are acknowledged with gratitude. The authors also wish to express their gratitude to Union Giken Co., Osaka, Japan for assistance in the construction of the apparatus.

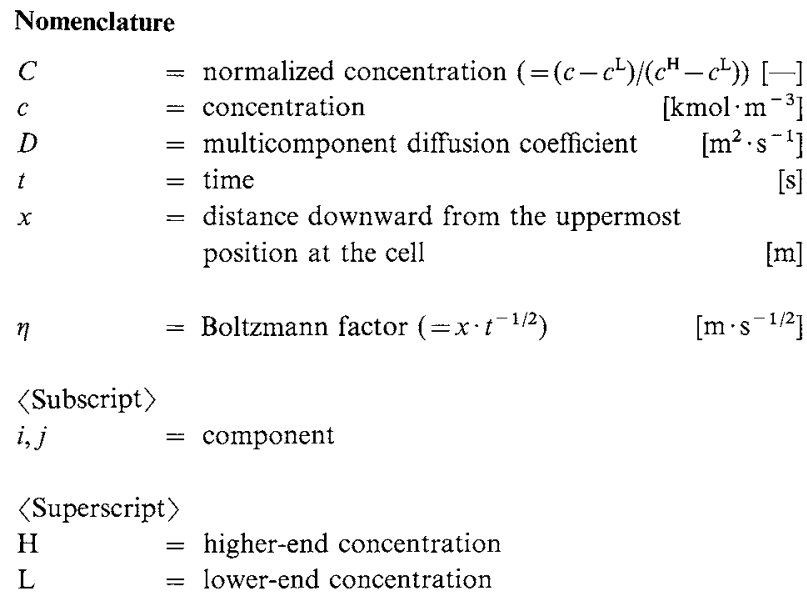

\section{Literature Cited}

1) Coulson, C. A., J. T. Cox, A. G. Ogston and J. St. L. Philpot: Proc. Roy. Soc. (London), A192, 382 (1948).

2) Cussler, E. L.: "Multicomponent Diffusion," Elsevier Sci. Pub. Co., Amsterdam (1976).

3) Eversole, W. G. and T. B. Dowghty: J. Phys. Chem., 41, 663 (1937).

4) Gösting, L. J., E. M. Hanson, G. Kegels and M. S. Morris: Rev. Sci. Instrum., 20, 209 (1949); Dunlop, P. J. and L. J. Gösting: J. Am. Chem. Soc., 77, 5238 (1955); Fujita, H. and L. J. Gösting: J. Am. Chem. Soc., 78, 1099 (1956).

5) Graff, R. A. and T. C. Drew: Ind. Eng. Chem. Fundam., 7, 490 (1968).

6) Kamiwano, M., M. Hasegawa and K. Ohya: Kagaku Kogaku Ronbunshu, 4, 653 (1978).

7) Linhart, T: Z. Phys., 105, 45 (1937).

8) Neurath, H.: Science, 93, 431 (1941); Chem. Revs., 30, 357 (1942).

9) Tanigaki, M., K. Kondo, M. Harada and W. Eguchi: J. Phys. Chem., 87, 586 (1983).

10) Tanigaki, M., S. Machida, M. Harada and W. Eguchi: $J$. Chem. Eng. Japan, 16, 257 (1983). 